\title{
Hypertension in aortic stenosis: More questions than answers
}

\author{
Ewa Orłowska-Baranowska \\ Institute of Cardiology, Department of Valvular Heart Diseases, Warsaw, Poland
}

\begin{abstract}
Although hypertension and aortic stenosis are the most common cardiovascular diseases, the impact of hypertension on the natural history of aortic stenosis, the structure and function of the left ventricle, the assessment of valve defect severity and its progression are not fully understood. Hypertension not only can modify the exploratory findings of aortic stenosis, but may also interfere with the assessment of severity, and even have an impact on patients outcome. In the absence of specific cohort studies, the nature of the association between aortic stenosis and high blood pressure is not clear and the published results are often contradictory. Unknown is the true frequency of both conditions, the rules of diagnosis and the treatment itself. (Cardiol J 2013; 20, 1: 4-10)
\end{abstract}

Key words: aortic stenosis, hypertension

\section{Introduction}

Aortic stenosis (AS) is the most common valvular heart disease in adults. It is the third cause of cardiovascular disease after hypertension and coronary artery disease. Nowadays, AS is generally caused by a progressive calcification of the aortic leaflets and due to increasing life expectancy in the general population, this form of valvular heart disease is now regarded as the coming plague of the $21^{\text {st }}$ century [1].

Although hypertension and AS are the most common cardiovascular disease, the impact of hypertension on the natural history of AS, the structure and function of the left ventricle (LV), the assessment of the degree of defect and its progression are not fully understood [2]. The problems associated with coexistence of both diseases are not a frequent subject of studies, what is more, the published results are often contradictory. Unknown is the true frequency of the coexistence of both conditions, the rules of diagnosis and the treatment.
Because of the prolongation of life span, we should expect an increase in the incidence of AS in patients with hypertension as well as the reverse situation - more cases of hypertension in the group of patients with AS.

\section{What is the prevalence of hypertension in patients with aortic stenosis?}

The first question without answer is the frequency of coexistence of both diseases in population. The true coexistence of AS and hypertension is difficult to estimate [3-13]. Only few population-based studies on a representative group that would allow estimate the frequency of the coexistence of both diseases were carried out. The assessment of diagnoses with which the patients were discharged from hospitals in 1993-1999 in Ireland (3.39 million cases) estimated that hypertension was the first or subsequent diagnosis in $6.2 \%$ of all patients, and AS in $0.33 \%$ - both diseases were observed in $0.07 \%$ of cases. Hypertension was found in $21 \%$ of patients

Address for correspondence: Prof. Ewa Orłowska-Baranowska, Department of Valvular Heart Diseases,

Institute of Cardiology, ul. Alpejska 42, 04-628 Warszawa, Poland, tel: + 48, 22343 44 47, fax: +48 223434509 ,

e-mail: eorlowska@ikard.pl

Received: 07.08.2012

Accepted: 08.10.2012 
with AS, but AS only in 1.1\% of patients with hypertension [12].

Most information about the coexistence of both diseases can be found in the studies concerning the patients referred for surgical treatment in case of symptomatic AS or observed in case of asymptomatic or insignificant defect. In these incidence of hypertension in patients with AS varies from $33 \%$ to $90 \%[2,4,13-15]$. The highest incidence described in the literature was reported in symptomatic patients with moderate/significant AS, in whom myocardial fibrosis was observed prospectively. Hypertension was found in $80 \%$ of patients with moderate and $90 \%$ with significant AS with low transvalvular pressure gradient with preserved LV systolic function - the majority of these patients were older than 70 years [15]. In patients in whom the effect of cholesterol-lowering drugs on the progression of AS was observed, hypertension was found more often than in the population without heart defects [16]. Also comparison of the frequency of hypertension among men and women with AS provide conflicting results. In some studies hypertension occurs in patients with AS more frequently among women (46\%) than men (29\%) [17].

There is no doubt that in recent years, due to the aging population, prolonged survival and increased accessibility to modern methods of diagnosis, frequency of diagnoses of hypertension in patients with AS significantly increased [18]. Both diseases may have common pathophysiological mechanism, may be coincidence of common diseases or may be closely linked. Moreover, there is the evidence that hypertension is an independent factor in the development of degenerative forms of AS in elderly $[19,20]$.

\section{Does hypertension affect the diagnosis of aortic stenosis?}

Hypertension is a disease in which symptoms usually occur late, at the onset of organ damage. It is often detected during random pressure measurements. Aortic stenosis is also asymptomatic for many years and it happens that diagnosis of valve disease is made on the basis of typical murmur or ECHO examination performed of other indications.

In case of a significant defect and appearance of typical symptoms of AS (angina, symptoms of low cardiac output or heart failure) indication for surgery is mandatory [21].

In patients with AS and hypertension, greater hypertrophy, increased wall thickness and LV mass may lead to the development of symptoms, partic- ularly shortness of breath at a lower stage of valve defect, lower transvalvular gradients and higher aortic valve area [22]. Here another question about the treatment of such patients arises - recommendations of Cardiological Societies do not contain guidance on these particular patients. There is no answer to the question of whether such patients should be operated earlier? If not - what parameters are the best to follow-up in this case in the future? Are we to manage patients for surgery when the gradient increases and the valve area significantly decrease, and in the meantime to treat patients conservatively despite typical symptoms?

On the other hand, in advanced AS, the diagnosis of hypertension may be easily overlooked most of the authors point out that the value of increased blood pressure with the progress of AS decreases. What is more, the older textbooks of cardiology even pay attention to the fact that increased pressure in patients with significant AS is very rare [23, 24].

Another problem is related to the physical examination. The auscultatory phenomena in AS are highly characteristic. It should be emphasized that the cardiac auscultation is the cheapest and the most diagnostic method, yet, unfortunately, it is very often neglected in fascination of state-of-the art techniques. The heart murmur of AS is a typical crescendo-decrescendo (diamond-shaped) ejection murmur with no heard second tone. The murmur is sometimes accompanied by a systolic thrill. It is usually harsh, often even musical, and is most audible over the aortic area. The radiation of the murmur is quite extensive over both carotid arteries, left supra- and intraclavicular fosse and jugular fossa. It may also be heard over the back. Sometimes the murmur becomes quiet over the middle portion of the sternum and reappears at the apex. In the elderly, this may be the only area where it is heard $[21,23,25,26]$.

All the more surprising is the fact, that in those patients with hypertension, who have been under the care of doctors for many years; AS is often diagnosed at a very advanced stage, when clinical symptoms appear. By this time, unfortunately, in hypertensive patients murmur is treated as irrelevant ejection murmur and this may lead to delay in the diagnosis of disease. It happens sometimes, that AS is recognized with the change of physician.

On the other hand, this condition can also be explained by the fact that hypertension can modify the physical examination findings of AS, particularly in the elderly patients. The characteristic exploratory findings of AS are all influenced by peripher- 
al aortic impedance, valve reflections, poststenosic aortic dilatation and vessel stiffness. Therefore, coexisting hypertension may result in a carotid pulse with a rapid upstroke and normal amplitude, as well, as in a diminished murmur or a near normal second cardiac sound [3]. It should be also remembered that in older patients location of the murmur of AS is unusual - in 25\% the murmur may be heard only at the apex $[3,11,26]$.

Because of this limitation, the echocardiographic examination should never be obviated if a systolic murmur is heard, or if AS is suspected in a hypertensive patient.

\section{What is the effect of hypertension on echocardiographic assessment of aortic stenosis?}

Echocardiography confirms the diagnosis and the severity of AS. The examination should include: the measurement of blood flow velocity through the valve (Vmax), the definition of the pressure gradients $(\mathrm{PG})$ through the valve (peak PG and mean PG), the calculation of the aortic valve area (AVA), AVA indexed in relation to the body surface area (AVA//BSA) and the parameters describing the degree of left ventricular hypertrophy (LVH) and LV function [21, 26-29]. The morphology of the valve is also important (presence of significant calcifications). Hemodynamically significant AS is characterized by: AVA $<1 \mathrm{~cm}^{2}$, AVA/BSA $<0.6 \mathrm{~cm}^{2} / \mathrm{m}^{2}$, Vmax $>4 \mathrm{~m} / \mathrm{s}$ and mean $\mathrm{PG}>40 \mathrm{~mm} \mathrm{Hg}$.

Theoretically, hypertension can lead to increased peripheral resistance, it may also reduce the vulnerability of blood vessels, worsening LV function and cause changes in flow through the aortic valve $[7,30]$. In an experimental model, in vitro, echocardiographic and hemodynamic assessment of the effect of hypertension on the AVA and transvalvular flow parameters showed no flow changes secondary to changes in afterload [29]. On the other hand, significant changes in afterload caused by drugs or physical activity can modify the LV function, the transvalvular gradient and the volume flow [31]. This effect was confirmed in vivo in a porcine heart model. Hypertension leads to lower the transvalvular gradients - explained the mechanism of inheritance dependency of the gradient flow associated with increased systemic resistance $[11,24$, 32]. The effects of hypertension on the assessment of severity of AS in these experimental researches were focused on the influence of sudden changes in load (pressure) - as we know the impact of he- modynamic changes caused by chronic hypertension may be different [21].

Some authors suggest that the significance of valve stenosis may be masked by coexisting hypertension, especially by sudden changes and that the defect may be underestimated. Hence the severity of AS at high blood pressure should be interpreted with caution. In the ESC (2012) recommendation, in the part describing the echocardiographic assessment of AS, there is only one sentence informing that when hypertension is present, the severity should be reassessed when the patient is normotensive [21].

The recommendations of EAE/ASE (2009) include a few sentences about the measurement in patients with hypertension [34]. The experts note that "although a recent in vitro study has demonstrated that systemic pressure may not directly affect gradient and valve area measurements, increasing LV pressure load may cause changes in ejection fraction and flow. The presence of hypertension may therefore primarily affect flow and gradient but less AVA measurements. Nevertheless, evaluation of AS severity with uncontrolled hypertension may not accurately reflect disease severity. Thus control of blood pressure is recommended before echocardiographic evaluation, whenever possible". The experts stress that the echocardiographic report should always include a blood pressure measurement recorded at the time of the examination.

\section{Left ventricular hypertrophy in aortic stenosis: How much important is hypertension?}

Aortic stenosis, like hypertension is a gradually progressive disease, asymptomatic for many years of the clinical course - both are examples of diseases that cause LV overload and lead to LVH. However, the response to these two conditions does not proceed in the same way.

Aortic stenosis creates an obstruction to blood flow from the LV to the aorta which lead to LV pressure overload hypertrophy. The increase in pressure in the LV, myocardial hypertrophy and prolonged ejection time are basic compensatory mechanisms of AS, which make asymptomatic course of the disease for many years [21]. The LV adapts to elevated systolic blood pressure by means of concentric hypertrophy, which leads to thickening of the walls without increasing the LV cavity [35].

Left ventricular hypertrophy in arterial hypertension, in response to different types of overload and LV geometry change, in the case of high blood pressure may take the form of concentric remodel- 
ing, concentric or eccentric hypertrophy [35-37]. The adaptation of the LV is modified on the one hand the stage of hypertension, and type of medication efficacy and its duration, and, on the other, different clinical or hemodynamic factors - not without significance is the influence of genetic factors. The changes usually occur in $30-50 \%$ of patients, most of whom demonstrate eccentric hypertrophy, a different type of hypertrophy usually found in AS [36, 37].

In case of AS and hypertension we are dealing with two diseases, which generate $\mathrm{LVH}$ - in case of AS it is concentric hypertrophy and in case of hypertension - volume, pressure or mixed overload is possible - in practice it is difficult to separate the effect associated with both diseases. Aortic stenosis usually leads to concentric remodeling or concentric hypertrophy, regardless of the presence or not of hypertension [2, 4, 22].

The effect of hypertension on the size of LVH with coexisting hypertension is rarely described in the literature - less than it would indicate the frequency of coexistence of both diseases $[2,4,14,22$, $24,30]$. Some authors pointed out the significant effect of hypertension on the degree of hypertrophy, others did not show any difference in the degree of remodeling in the coexistence of these two conditions $[2,14,22,24,30]$. In the absence of published data which describe the impact of hypertension on electrocardiography evaluation of $\mathrm{LVH}$ in patients with AS, we apply the same electrocardiographic criteria as in isolated $\mathrm{LVH}$.

In a large group of patients (1720 patients from 173 European centers) with asymptomatic AS $(\operatorname{Vmax} \geq 2.5 \mathrm{~m} / \mathrm{s}$ and $\leq 4 \mathrm{~m} / \mathrm{s}$ ) enrolled in a study evaluating the impact of simwastatin and ezetimibe on the course of valve disease, the echocardiographic results were compared among the patients with/ /without coexistence of hypertension. In patients with hypertension there were more female patients, they were older and more obese. Compared to patients without high blood pressure, LVH, relative wall thickness ratio and wall thickness were observed more frequently - but what is interesting, eccentric hypertrophy was the most frequently observed type of hypertrophy, both in the group with hypertension and without it (concentric hypertrophy and concentric remodeling was recognized only in $8 \%$ in both) [14, 38].

We do not know the answer to the question which disease affects more on the LVH, arterial hypertension or AS? Garcia et al. [6] in the experimental model proved that in mild/moderate AS hypertension was a dominant factor in the remodeling of the LV, while in a significant stenosis effect of hypertension was less marked - the impact of AS far exceeded the impact of hypertension [6]. In AS changes are initially related to increased wall thickness, while the volume of the cavity does not change; in case of the coexistence of both diseases the dominant influence of hypertension determines the type of hypertrophy [37]. These studies confirmed the observations of a group of patients with hypertension and AS [4]. Moreover, the impact of hypertension on LVH was more marked in women [35, 39-42].

Asymmetric septal hypertrophy is usually seen in patients with hypertrophic cardiomyopathy. In people with hypertension, asymmetric septal hypertrophy is sometimes recognized in early stages of the LV adaptation. In patients with AS asymmetrical hypertrophy affects the extent of surgery. In the multivariate analysis performed in asymptomatic patients with AS arterial hypertension was the independent risk factor of asymmetric septal hypertrophy, regardless of severity of the defect [43]. From a theoretical point of view, LVH in AS is a beneficial compensatory response to excessive afterload, aimed at normalization of systolic stress in the wall of the LV [37]. However, for high blood pressure is less favorable the prognosis in patients who develop LVH compared to those with the normal LV geometry. In this group a higher mortality, higher incidence of coronary syndromes, ventricular arrhythmias and sudden cardiac death are observed. The risk of cardiovascular complications increases with the degree of LVH. Type of LV remodeling in hypertension is also associated with increased cardiovascular complications $-11 \%$ of the complications encountered in patients with normal LV, $15 \%$ when the current is concentric remodeling, $23 \%$ in patients with eccentric hypertrophy and $31 \%$ of the states concentric hypertrophy [44].

It seems that the coexistence of these two entities, which often results in higher $\mathrm{LVH}$, is associated with the increased risk of cardiovascular complications greater than in case of defects of isolated hypertension [35].

\section{Aortic valve replacement: When to perform in patients with aortic stenosis and hypertension?}

According to the current ESC and ACC/AHA guidelines, a decision to perform AVR should be based on two criteria: diagnosis of a severe AS (AVA $<1.0 \mathrm{~cm}^{2}$, AVA/BSA $<0.6 \mathrm{~cm}^{2} / \mathrm{m}^{2}$, mean $\mathrm{PG}>40 \mathrm{~mm} \mathrm{Hg}$ with preserved LV systolic function) and presence of symptoms [21,26]. There are often 
discrepancies between the severity of the stenosis and the symptomatic status. Some patients become symptomatic although they have only moderate AS, whereas others remain symptomatic despite the presence of severe stenosis. A patient having a moderate AS and concomitantly a moderate hypertension may have indeed higher LV afterload and more LVH than a patient with severe AS and normal blood pressure [6]. When AS is accompanied by hypertension, the LV faces a double burden valvular and vascular, so symptoms may occur earlier, before echocardiographic parameters will indicate significant AS.

The recommendations don't show any guidance on how to handle this case. In case of asymptomatic AS in the recommendations of the ESC class IIb indication for surgery is important LVH ( $>15 \mathrm{~mm}$ ), not caused by hypertension. As mentioned above, in case of a substantial defect and hypertension, stenosis is the strongest factor influencing LVH - maybe also in this group of patients the indications for surgical treatment should be considered earlier - to these question there is no answer. In recent years, at congresses and in pages of the cardiology literature the proceedings (whether treated by surgery) in patients with AS, in whom the preserved LV systolic function and significantly reduced valve area is not adequately recorded with high PG (low flow, low gradient AS with preserved ejection fraction) have been discussed. This group includes patients with small left chambers, and a large muscle hypertrophy, especially women - is not this just the effect of the impact of concomitant hypertension?

\section{How to treat hypertension in patients with aortic stenosis?}

As mentioned above, the effective treatment of symptomatic, significant AS is aortic valve replacement. Conservative treatment of advanced stage in case of contraindications to surgery or lack of patient consent is very difficult. Usually, it is effective for a very short period of time. Often, it may be harmful [21]. Even the severe valve defect, without symptoms, does not need pharmacological treatment.

On the other hand, even moderately, and poorly controlled hypertension can significantly accelerate and enhance LVH and reduce the benefits associated with the valve surgery in the future $[6,44]$. The recommendations of the ESC experts have pointed out that in case of significant AS hypertension should be treated with caution. The negative inotropic drugs such as beta-blockers should be avoid- ed. There is also concern that vasodilatators can lead to reduction in the coronary perfusion pressure [21].

Furthermore, the best drug for lowering blood pressure in the presence of an AS has not been established. A specific potential benefit has been suggested for the inhibitors of angiotensin converting enzyme (ACE), since ACE activity has been involved not only in unfavorable $\mathrm{LV}$ remodeling response, but also in the progression of valve deterioration. ACE inhibitors, that prolong life in most cases of congestive heart failure, like other vasodilatators, has been classically considered contraindicated in AS, because of their potentially hazardous effect or related to the risk of decreasing coronary perfusion pressure in this population of patients and may exacerbate symptoms. Cardiac output in severe AS can not be increased because of stenotic valve. Decrease in peripheral resistance increases the transvalvular gradient, leads to a drop in blood pressure, which can be fatal. The recent retrospective studies, although not planned to assess the safety of this drug in AS, showed that the daily practice of ACE inhibitors is widely used, because of concomitant hypertension and is well tolerated. In the SCOPE-AS study symptomatic patients with significant stenosis and normal LV systolic function, who were not candidates for surgical treatment, were randomized to the treatment with enalapril or placebo. The drug was well tolerated, although patients with poor LV function presented a tendency to hypotension more often. Similarly, the administration of ACE inhibitors in patients with moderate AS, despite lowering blood pressure and increase of the PG, did not result in deterioration of exercise tolerance and clinical symptoms. These observations suggest that ACE inhibitors may be used with caution in the treatment of hypertension in patients with AS. If they had been used before the diagnosis of AS with heart failure, hypertension or diabetic nephropathy and had been well tolerated by the patient, they should not be discontinued [21, 45-48]. Diuretic in AS should be used only in volume overload. The excessive preload reduction may lead to deterioration of the LV filling, reduced cardiac output and blood pressure. Patients with significant AS are at risk for the occurrence of these adverse events [21, 49].

\section{What is the hypertension's influence on early and late results of the surgical treatment of aortic stenosis?}

Despite such frequent coexistence of both diseases little is known about the impact of hypertension on early and late results of the surgical treat- 
ment and the pharmacological management after aortic valve replacement. The only effective treatment of AS is aortic valve replacement $[21,26]$. The effect of concomitant hypertension on long-term fate of patients after aortic valve replacement is not fully understood [50-57].

The implantation of an artificial valve eliminates the systolic overload associated with AS, but the load associated with hypertension remains [58]. This explains why patients with hypertension may still have important LVH despite the valve replacement or had worse LVH reduction after the surgery.

Here arises another question about monitoring the effectiveness of the pharmacological treatment of hypertension in patients after aortic valve replacement. We don't know, if like in the general population, the treatment of hypertension in this group is well controlled; there is lack of studies on that subject, beyond the announcement many years ago, in which hypertension was treated correctly only in 8 of 43 patients [57].

Another question concerns the impact of hypertension on the rate of biological prostheses degeneration - there is a lack of data on this subject, what will be the impact of hypertension on longterm observation of patients after transaortic valve implantation [19, 20].

\section{Summary}

Systemic hypertension, like AS, is nowadays a frequent finding due to increase in life expectancy and aging of population. It is expected that the diagnosis of AS in patients with hypertension will occur more frequently.

The more research is needed that will clarify the answers to the questions above, especially with the use of modern diagnostic methods, the dynamic growth of which was seen in recent years [58-60]. These facts need to be considered for an adequate diagnosis and management of patients with AS.

It is also important to note that hypertension, on one hand, is well established risk for cardiovascular events in the general population, and, on the other, is one of the factors considered to be escalating progression of defects but the treatment in the coexistence of both diseases is not easy.

\section{Conflict of interest: none declared}

\section{References}

1. D'Arcy JL, Prendergast BD, Chambers JB, Ray SG, Bridgewater B. Valvular heart disease: the next cardiac epidemic. Heart, 2011: 97: 91-93.
2. Antonini-Canterin F, Huang G, Cervesato E et al. Symptomatic aortic stenosis: Does systemic hypertension play an additional role? Hypertension, 2003; 41: 1268-1272.

3. Bermejo J. The effect of hypertension on aortic valve stenosis. Heart, 2005; 91: 280-282.

4. Hueb JC, Joao TR, Goi VM et al. Impact of hypertension on ventricular remodeling in patients with aortic stenosis. Arq Bras Cardiol, 2011; 97: 254-258.

5. Jolobe OM. Systolic hypertension is also the neglected stepsister of aortic stenosis. Am J Med, 2008; 121: e21.

6. Garcia D, Pibarot P, Kadem L, Durant LG. Respective impacts of aortic stenosis and systemic hypertension on left ventricular hypertrophy. J Biomech, 2007; 40: 972-980.

7. Kaden JJ, Haghi D. Hypertension in aortic valve stenosis: A trojan horse. Eur Heart J, 2008; 29:1934-1935.

8. Faggiano P, Frattini S, Zilioli V et al. Prevalence of comorbidities and associated cardiac diseases in patients with valve aortic stenosis. Potential implications for the decision-making process. Int J Cardiol, 2011; 2012; 159: 94-99.

9. Pagé A, Dumesnil JG, Clavel MA, Chan KL et al. Metabolic syndrome is associated with more pronounced impairment of left ventricle geometry and function in patients with calcific aortic stenosis: A substudy of the ASTRONOMER (Aortic Stenosis Progression Observation Measuring Effects of Rosuvastatin). J Am Coll Cardiol, 2010; 55: 1867-1874.

10. de Simone $\mathrm{G}$. The difficult clinical management of the combination of hypertension with aortic stenosis. J Hypertens, 2010; 28: 234-236.

11. Kadem L, Dumesnil JG, Rieu R, Durand LG, Garcia D, Pibarot P. Impact of systemic hypertension on the assessment of aortic stenosis. Heart, 2005; 91: 354-361.

12. Pate GE. Association between aortic stenosis and hypertension. J Heart Valve Dis, 2002; 11: 612-614.

13. Orlowska-Baranowska E, Rawczyńska-Englert I. Risk factors for coronary artery stenosis in valvular heart disease. J Heart Valve Dis, 1998; 7: 586-589.

14. Rieck AE, Cramariuc D, Staal EM et al. Impact of hypertension on left ventricular structure in patients with asymptomatic aortic valve stenosis (a SEAS substudy). J Hypertens, 2010; 28: 377-383.

15. Herrmann S, Störk S, Niemann M et al. Low-gradient aortic valve stenosis myocardial fibrosis and its influence on function and outcome. J Am Coll Cardiol, 2011; 58: 402-412.

16. Rossebo A, Pedersen T, Skjaerpe T, Mitchel Y, Larsen V. Design of the simvastatin and ezetimibe in aortic stenosis (SEAS0 study. Atherosclerosis, 2003; 4 (suppl.): 253-257

17. Higgins J, Jamieson WR, Benhameid O et al. Influence of patient gender on mortality after aortic valve replacement for aortic stenosis. J Thorac Cardiovasc Surg, 2011; 142: 595-601.

18. McLean RC, Briggs AH, Slack R et al. Perioperative and long-term outcomes following aortic valve replacement: A population cohort study of 4124 consecutive patients. Eur J Cardiothorac Surg, 2011; 40: 1508-1514.

19. Lindroos S, Kupari M, Heikkila J et al. Prevelence of aortic valve abnormalities in the elderly: An echocardiographic study of random population sample. J Am Coll Cardiol, 1993; 21: 1220-1225.

20. Stewart BF, Siscovick D, Lind BK et al. Clinical factors associated with calcific aortic valve disease. Cardiovascular health study. J Am Coll Cardiol, 1997; 29: 630-634.

21. Valhanian A. Alfieri O, Andreotti F et al. Guidelines on the management of valvular heart disease (version 2012): The Joint Task Force of the Management of Valvular Heart Disease of the European Society of Cardiology (ESC) and the European Association for Cardio-Thoracic Surgery (EACTS). Eur J Cardiothorac Surg, 2012; 42: S1-S44.

22. Antonini-Canterin F, Huang G, Cervesato E et al. Reliability of new and old Doppler echocardiographic indexes of the severity of aortic stenosis in patients with a low cardiac output. Ital Heart J, 2002; 3: 248-255. 
23. Hoffman M, Rydlewska-Sadowska W, Rużyło W. Wady serca. PZWL, Warszawa 1989.

24. Chambers J. Can high blood pressure mask severe aortic stenosis. J Heart Valve Dis, 1998; 8: 277-278.

25. Das P, Pocock C, Chambers W. The patient with systolic murmur: severe aortic stenosis may be missed during cardiovascular examination. QJM, 2000; 93: 685-688.

26. Bonow Bonow RO, Carabello B, deLeon AC et al. ACC/AHA guidelines for the management of patients with valvular heart disease: A report of the American College of Cardiology/American Heart Association task force on practice guidelines (committee on management of patients with valvular heart disease). J Am Coll Cardiol, 1998; 32: 1486-1588.

27. Zoghbi WA, Farmer KL, Soto JG, Nelson JG, Quinones MA. Accurate noninvasive quantification of stenotic aortic valve area by Doppler echocardiography. Circulation, 1986; 73: 452-459.

28. Wong RC, Yeo T. Stroke-work Loss underestimates hemodynamic significance of aortic stenosis in patients with hypertension. Echocardiography, 2007; 24; 673-676.

29. Mascherbauer J, Fuchs C, Stoiber M et al. Systemic pressure does not directly affect pressure gradient and valve area estimates in aortic stenosis in vitro. Eur Heart J, 2008; 29: 2049-2057.

30. Linhartova K, Filipovsky J, Cerbak R, Sterbakova G, Hanisova I, Beranek V. Severe aortic stenosis and its association with hypertension: analysis of clinical and echocardiographic parameters. Blood Press, 2007; 16: 122-128.

31. Little SH, Chan KL, Burwash IG. Impact of blood pressure on the Doppler echocardiographic assessment of the severity of aortic stenosis. Heart, 2005; 91: 354-361.

32. Laskey WK, Kussmaul WG, Noordergraaf A. Valvular and systemic arterial hemodynamics in aortic valve stenosis. Circulation, 1995; 92: 1473-1478.

33. Pibarot P, Dumesnil JG. Assessment of aortic stenosis severity: Check the value but don't forgrt the arteries. Heart, 2007; 93: 780-782.

34. Baumgartner H, Hung J, Bernejo J et al. Echocardiographic assessment of valve stenosis: EAE/ASE recommendations for clinical practice. J Am Society Echocardiog, 2009; 22: 1-20.

35. Gerdts E. Left ventricular structure in different types of chronic pressure overload. Eur Heart J, 2008; 10 (suppl. E): E23-E30.

36. Devereux RB, Reichek N. Echocardiographic determination of left ventricular mass in man: Anatomic validation of the method. Circulation, 1977; 55: 613-618.

37. Ganau A, Devereux RB, Roman MJ et al. Patterns of left ventricular hypertrophy and geometric remodeling in essential hypertension. J Am Coll Cardiol, 1992; 19: 1550-1558.

38. Cramariuc D, Rieck AE, Staal EM et al. Factors influencing left ventricular structure and stress-corrected systolic function in men and women with asymptomatic aortic valve stenosis (a SEAS Substudy). Am J Cardiol, 2008; 101: 510-515.

39. Agabiti-Rosei E, Salvetti M. Gender differences in the regression eletrocardiographic left ventricular hypertrophy during antihypertensive therapy. Hypertension, 2008; 52: 59-60.

40. Regitz-Zagrosek V, Oertelt-Prigione S, Seeland U, Hetzer R. Sex and gender differences in myocardial hypertrophy and heart failure. Circ J, 2010; 74: 1265-1273.

41. Villar AV, Liano M, Cobo M et al. Gender differences of echocardiographic and gene expression patterns in human pressure overload left ventricular hypertrophy. J Mol Cell Cardiol, 2009; 46: 526-535.

42. Verdecchia P, Schillaci G, Boldrini F, Guerrieri M, Porcellati C. Sex, cardiac hypertrophy and diurnal blood pressure variations in essential hypertension. J Hypertens, 1992; 10: 683-692.
43. Tuseth N, Cramariuc D, Rieck AE, Wachtell K, Gerdts E. Asymmetric septal hypertrophy: A marker of hypertension in aortic stenosis (a SEAS substudy). Blood Press, 2010; 19: 140-144.

44. Koren MJ, Devereux RB, Casale PN, Savage DD, Laragh JH. Relation of left ventricular mass and geometry to morbidity and mortality in uncomplicated essential hypertension. Ann Intern Med, 1991; 114: 345-352.

45. Aurigemma GP, Keaney JF Jr. Renin-angiotensin system inhibition for aortic stenosis “A II, Bruté?”. J Am Coll Cardiol, 2011; 58: 577-580.

46. Shavelle DM. Are angiotensin converting enzyme inhibitors beneficial in patients with aortic stenosis? Heart, 2005; 91: 1257-1259.

47. Nadir MA, Wei L, Elder DH et al. Impact of renin-angiotensin system blockade therapy on outcome in aortic stenosis. J Am Coll Cardiol, 2011; 58: 570-576.

48. Jimenez-Candil J, Bermejo J, Yotti R et al. Effects of angiotensin converting enzyme inhibitors in hypertensive patients with aortic valve stenosis: A drug withdrawal study. Heart, 2005; 91: 1311-1318.

49. Newby DE, Cowell SJ, Boon NA. Emerging medical treatments for aortic stenosis: Statins, angiotensin converting enzyme inhibitors, or both? Heart, 2006; 92: 729-734.

50. Ali A, Patel A, Ali Z et al. Enhanced left ventricular mass regression after aortic valve replacement in patients with aortic stenosis is associated with improved long-term survival. J Thorac Cardiovasc Surg, 2011; 142: 285-291

51. Kalavrouziotis D, Li D, Buth KJ, Légaré JF. The European System for Cardiac Operative Risk Evaluation (EuroSCORE) is not appropriate for withholding surgery in high-risk patients with aortic stenosis: A retrospective cohort study J Cardiothorac Surg, 2009; 14: 32-34.

52. Kobayashi KJ, Williams JA, Nwakanma LU et al. EuroSCORE predicts short- and mid-term mortality in combined aortic valve replacement and coronary artery bypass patients. J Card Surg, 2009; 24 : 637-643.

53. Mack MJ. Risk scores for predicting outcomes in valvular heart disease: How useful? Curr Cardiol Rep, 2011; 13: 107-112.

54. Di Eusanio M, Fortuna D, De Palma et al. Aortic valve replacement: Results and predictors of mortality from a contemporary series of 2256 patients. J Thorac Cardiovasc Surg, 2011; 141: 940-947.

55. Lund O, Emmersten K, Dorup et al. Regression of left ventricular hypertrophy during 10 years after valve replacement for aortic stenosis is related to the preoperative risk profile. Eur Heart J, 2003; 24: 1437-1446.

56. Sharma UC, Barenbrug P, Pokharel S, Dassen WR, Pinto YM, Maessen JG. Systematic review of the outcome of aortic valve replacement in patients with aortic stenosis. Ann Thorac Surg, 2004; 78: 90-98.

57. McGinn S, White PD. Clinical observation of aortic stenosis. Am J Med Sci, 1934; 188: 1-15.

58. Yarbrough WM, Mukherjee R, Ikonomidis JS, Zile MR, Spinale FG. Myocardial remodeling with aortic stenosis and after aortic valve replacement: Mechanisms and future prognostic implications. J Thorac Cardiovasc Surg, 2011; 2012; 143: 656-664.

59. Puntmann VO, Jahnke C, Gebker R et al. Usefulness of magnetic resonance imaging to distinguish hypertensive and hypertrophic cardiomyopathy. Am J Cardiol, 2010; 106: 1016-1022.

60. Rudolph A, Abdel-Aty H, Bohl S et al. Noninvasive detection of fibrosis applying contrast-enhanced cardiac magnetic resonance in different forms of left ventricular hypertrophy relation to remodeling. J Am Coll Cardiol, 2009; 53: 284-2891. 